\title{
Valve-sparing aortic root replacement in bicuspid aortic valves: A reasonable option?
}

\author{
Diana Aicher, MD \\ Frank Langer, $\mathrm{MD}^{\mathrm{a}}$ \\ Anke Kissinger ${ }^{\mathrm{a}}$ \\ Henning Lausberg, MD \\ Roland Fries, MD ${ }^{\mathrm{b}}$ \\ Hans-Joachim Schäfers, MD
}

From the Department of Thoracic and Cardiovascular Surgery and the Department of Internal Medicine III, ${ }^{\mathrm{b}}$ University Hospitals Homburg, Homburg/Saar, Germany.

Read at the Eighty-fourth Annual Meeting of The American Association for Thoracic Surgery, Toronto, Ontario, Canada, April 25-28, 2004.

Received for publication April 18, 2004; revisions received June 4, 2004; accepted for publication June 21, 2004.

Address for reprints: Hans-Joachim Schäfers, MD, Department of Thoracic and Cardiovascular Surgery, University Hospitals Homburg, 66424 Homburg, Germany (E-mail: chhjsc@uniklinik-saarland.de).

J Thorac Cardiovasc Surg 2004;128:662-8 $0022-5223 / \$ 30.00$

Copyright $\odot 2004$ by The American Association for Thoracic Surgery

doi:10.1016/j.jtcvs.2004.06.035
Objectives: Aortic dilatation occurs in many patients with bicuspid aortic valves. We have added root replacement using the remodeling technique originally designed for tricuspid aortic valves to bicuspid aortic valve repair for treatment of the dilated root. We compared the results of remodeling in bicuspid aortic valves with those in tricuspid aortic valves.

Methods: From October 1995 through January 2004, 60 patients underwent root remodeling for bicuspid aortic valves (group A), and 130 patients underwent root remodeling for tricuspid aortic valves (group B). Correction of cusp prolapse was more often performed in group A (group A, 50/60; group B, 47/130; $P<.0001$ ). Transthoracic echocardiography was performed at 1 week, 6 and 12 months, and every year thereafter. Cumulative follow-up was 527 patient-years (mean, $2.9 \pm 2$ years).

Results: No patient died in group A. Hospital mortality in group B was 5\% (5/100; 95\% confidence interval, 1.6\%-11.3\%) after elective operations and 10\% (3/30; $95 \%$ confidence interval, $2.1 \%-26.5 \%$ ) after emergency operations. Mean systolic gradients were identical at 1 year (group A, $4.8 \pm 2.1 \mathrm{~mm} \mathrm{Hg}$; group B, $4.0 \pm 2 \mathrm{~mm}$ $\mathrm{Hg}$ ) and 5 years (group A, $4.5 \pm 2.3 \mathrm{~mm} \mathrm{Hg}$; group B, $3.9 \pm 2.2 \mathrm{~mm} \mathrm{Hg}$ ). Freedom from aortic regurgitation of grade 2 or higher at 5 years was $96 \%$ in group A and $83 \%$ in group B $(P=.07)$, and freedom from reoperation at 5 years was $98 \%$ in group $\mathrm{A}$ and $98 \%$ in group $\mathrm{B}(P=.73)$.

Conclusions: Valve-sparing aortic replacement with root remodeling can be applied to aortic dilatation and a regurgitant bicuspid aortic valve. Hemodynamic function and valve stability of a repaired bicuspid aortic valve are comparable with those seen in cases of tricuspid anatomy.

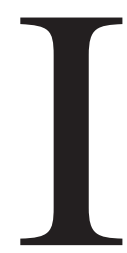

$\mathrm{n}$ the past 10 years, valve-sparing aortic replacement has become an alternative to composite replacement of the valve and aorta for regurgitant tricuspid aortic valve and aortic dilatation. ${ }^{1-5}$ Both remodeling of the aortic $\operatorname{root}^{1}$ and reimplantation of the aortic valve within a vascular graft ${ }^{3}$ have been applied, with technical variations. Both approaches avoid the complications of prosthetic heart valve replacement. Initially designed for regurgitant valves with preserved cusp morphology, valve preservation has also been combined with correction of cusp prolapse with good results. ${ }^{6}$

Bicuspid anatomy of the aortic valve is one of the main causes of aortic regurgitation, particularly in young individuals. In these patients there is concern regarding the use of pulmonary autografts. ${ }^{7}$ Although composite replacement with a mechanical prosthesis is a safe option, ${ }^{8}$ young patients are also more likely to 
experience cumulative prosthetic valve-related morbidity because of their long anticipated lifespan. In addition, anticoagulation might not be desired by individuals with an active lifestyle.

Isolated reconstruction of a regurgitant bicuspid valve has been performed with good early results. ${ }^{9,10}$ Midterm results, however, have been limited by a variable incidence of recurrent regurgitation. ${ }^{11,12}$ Dilatation of the aortic root has been reported as a risk factor for recurrence of regurgitation. ${ }^{11}$ Dilatation of the proximal aorta has been observed in up to $50 \%$ of individuals with bicuspid aortic valves. ${ }^{13,14}$ This has been found to be associated with histologic evidence of aortic wall pathology ${ }^{15}$ and is increasingly accepted as a justification for prophylactic aortic replacement. ${ }^{16}$

We have previously applied the technique of root remodeling in combination with valve repair for treatment of bicuspid aortic valves and proximal aortic dilatation. ${ }^{17}$ In a limited series we could demonstrate the feasibility of this combined approach. We now report midterm results in a larger patient cohort, with follow-up reaching up to 8 years, and compared these results with those of root remodeling in tricuspid aortic valves.

\section{Patients and Methods}

From October 1995 through January 2004, 190 patients (136 men and 54 women) were treated with remodeling of the aortic root for aortic regurgitation and proximal aortic dilatation. In 60 patients the aortic valve was bicuspid, and 130 patients had tricuspid valve anatomy. In all instances dilatation $(\geq 5 \mathrm{~cm}$, as determined by means of preoperative computed tomography) or dissection of the ascending aorta was present.

In all patients the chest was opened with a median sternotomy, and the patient was started on extracorporeal circulation with aortic and right atrial cannulation. In acute dissection the right femoral $(n=25)$ or right axillary $(n=9)$ artery was used for arterial cannulation. Cardioplegic arrest was induced by means of infusion of blood cardioplegia into the coronary ostia. The aortic root was inspected, and diameters of aortoventricular junction and sinotubular junction were measured with graded valve sizers. Root remodeling was chosen when the sinotubular diameter exceeded 33 to $35 \mathrm{~mm}$ and the aortoventricular diameter was $30 \mathrm{~mm}$ or less.

In all instances a Dacron graft was chosen that was 1 to $2 \mathrm{~mm}$ smaller than the diameter of the aortoventricular junction.

If the valve was tricuspid, the graft was tailored to create 3 symmetric neosinuses. The sinuses were excised, and the root was replaced with the graft, thus re-establishing a normal configuration. The aortic valve was carefully assessed for configuration and coaptation. In 47 instances relevant stretching of the free margin of 1 or 2 cusps with prolapse was found. This was corrected with commissural $(n=4)$ or central $(n=42)$ plicating sutures and in one instance with triangular resection of cusp tissue.

If the valve was bicuspid, the aortic sinuses were similarly excised. In the presence of a symmetric bicuspid valve $(n=2)$, symmetric root geometry was also present. In 58 instances congenital fusion between the right and left coronary cusps was found.
The root showed typical asymmetry, with a large noncoronary sinus and 2 rudimentary sinuses. The Dacron graft was tailored to accommodate the individual geometry of the root. In 5 patients an aortoventricular junction of $30 \mathrm{~mm}$ was treated with subcommissural plication.

Prolapse of the congenitally fused cusp was seen in 50 patients and corrected with commissural $(n=1)$ or central $(n=39)$ shortening sutures of the cusp margin after applying radial tension on the 2 main commissures $(n=40)$. Triangular resection of a median raphe was performed only if dense fibrotic tissue or calcifications prevented direct shortening $(n=20)$.

Gross evidence of prolapse was corrected before performing root remodeling. Monofilament suture material (5-0 or 6-0 Prolene) was used in all patients. In all instances aortic valve geometry was studied again for cusp prolapse after implantation of the vascular graft. Additional cusp plication sutures were then placed as was believed necessary to achieve correct cusp coaptation. The coronary ostia were implanted into the graft in standard fashion. If arch disease was present, replacement of the arch was performed during hypothermic circulatory arrest $\left(18^{\circ} \mathrm{C}-21^{\circ} \mathrm{C}\right.$ nasopharyngeal temperature). Concomitant cardiac procedures were performed as needed.

Intraoperative transesophageal echocardiography (HDI 3000; ATL Technologies, Hagen, Germany) was performed after weaning from cardiopulmonary bypass and with a diastolic blood pressure of $70 \mathrm{~mm} \mathrm{Hg}$, using vasopressors if necessary. A semiquantitative assessment of the degree of aortic valve regurgitation was performed with continuous-wave Doppler scanning by using the intensity and slope of the regurgitation signal. ${ }^{18}$ In addition, the width of the regurgitation jet in relation to the diameter of the left ventricular outflow tract was measured by means of color Doppler scanning. ${ }^{19}$ Systolic gradients were recorded with continuouswave Doppler scanning ${ }^{20}$.

Postoperatively, transthoracic echocardiography was performed for determination of aortic regurgitation and systolic gradients at 1 week, 6 and 12 months, and every 12 months thereafter.

Most patients $(n=168)$ were followed in our outpatient clinic, and the remaining patients $(n=22)$ were followed by their local cardiologists. The echocardiograms were performed by the same echocardiographers (DA, FL, and HL) and reviewed by an experienced cardiologist (RF). Video documentation of echocardiographies performed by local cardiologists was reviewed by the same experienced cardiologist (RF).

Follow-up ranged from 1 to 99 months and was complete in 189 of 190 patients. Cumulative follow-up was 527 patient-years (mean, $2.9 \pm 2$ years).

The patients were analyzed in 2 groups according to the anatomy of the aortic valve being bicuspid (group $\mathrm{A}, \mathrm{n}=60$ ) or tricuspid (group $\mathrm{B}, \mathrm{n}=130$ ).

Continuous data are expressed as means \pm SD and analyzed by using the Mann-Whitney $U$ test. Categoric variables are described by contingency tables and analyzed with the $\chi^{2}$ test. Corresponding $95 \%$ confidence intervals (CIs) are calculated by means of Sisa online statistical analysis. Kaplan-Meier estimates of freedom from reoperation and degree of aortic regurgitation were calculated with a commercially available software package (Prism; GraphPad Inc, San Diego, Calif). 
TABLE 1. Clinical data of $\mathbf{1 9 0}$ patients having undergone root remodeling in the presence of bicuspid or tricuspid aortic valve anatomy

\begin{tabular}{lccr}
\hline & BAV & TAV & P value \\
\hline $\mathrm{N}$ & 60 & 130 & \\
Age (y) & $53 \pm 12(30-74)$ & $64 \pm 14(3-86)$ & $>.001$ \\
Sex (M/F) & $50 / 10$ & $86 / 44$ & .015 \\
Aneurysm & 56 & 100 & .006 \\
Acute dissection & 4 & 30 & .006 \\
Concomitant procedures & & & \\
$\quad$ CABG & 8 & 35 & .037 \\
$\quad$ MVR & 1 & 8 & .18 \\
$\quad$ Aortic arch replacement & 14 & 87 & $<.001$ \\
$\quad$ Partial & 14 & 69 & .001 \\
$\quad$ Total & $106 \pm 33$ & 18 & .002 \\
Extracorporal circulation (min) & $79 \pm 13$ & $131 \pm 50$ & .001 \\
Myocardial ischemia (min) & $11 \pm 4$ & $90 \pm 23$ & .001 \\
Circulatory arrest (min) & $40 \pm 0.6(33-50)$ & $16 \pm 10$ & .042 \\
Sinotubular diameter (mm) & $27 \pm 0.2(24-34)$ & $41 \pm 0.6(33-65)$ & .407 \\
Aortoventricular diameter (mm) & $25 \pm 1.2(24-28)$ & $24 \pm 0.2(22-29)$ & .001 \\
Graft size (mm) & $25 \pm 1.5(18-28)$ & $<.001$ \\
\hline
\end{tabular}

$B A V$, Bicuspid aortic valve; TAV, tricuspid aortic valve; $C A B G$, coronary artery bypass grafting; $M V R$, mitral valve repair.

\section{Results}

There were significant differences between the groups, mostly related to different patient characteristics. Among patients with bicuspid aortic valves, patients were younger $(P<.001)$, fewer patients required emergency operations for acute dissection $(P=.006)$, and there was less need for arch replacement $(P<.001)$ and concomitant coronary bypass surgery $(P=.037$; Table 1$)$. Consequently, extracorporeal circulation $(P=.001)$, myocardial ischemia $(P=$ $.001)$, and circulatory arrest $(P=.04)$ times were shorter in the presence of bicuspid aortic valves. Myocardial ischemic times for isolated remodeling (ie, without concomitant cardiac surgery) were identical in group A ( $75 \pm 8$ minutes) and group B (77 \pm 11 minutes).

In-hospital mortality for all patients was $4.2 \%(8 / 190$; $95 \%$ CI, $1.8 \%-8.1 \%)$. No patient died after repair of a bicuspid aortic valve $(0 / 60 ; 95 \% \mathrm{CI}, 0.05 \%-6 \%)$. Of the 8 patients who died in group B $(6.2 \%$; 95\% CI, 3.2\%-12.7\%), $3 \mathrm{had}$ undergone operations for acute dissection. Mortality in group B was thus 5\% (5/100; 95\% CI, 1.6\%-11.3\%) after elective procedures and 10\% (3/30; 95\% CI, 2.1\%-26.5\%) after emergency procedures. The causes of death were mesenteric ischemia with sepsis and multiple organ failure $(\mathrm{n}=$ $5)$, cerebral ischemia $(n=1)$, pulmonary embolism $(n=1)$, and left ventricular failure $(\mathrm{n}=1)$. One patient in group A and 5 patients in group $\mathrm{B}$ required re-exploration for bleeding $(P=.42)$.

The preoperative degree of aortic regurgitation was 2.5 \pm 0.9 in group $\mathrm{A}$ and $2.8 \pm 0.8$ in group $\mathrm{B}(P=.24)$. This was reduced to $0.8 \pm 0.7$ in group $\mathrm{A}$ and $0.9 \pm 0.8$ in group $\mathrm{B}$ at discharge. The degree of regurgitation remained con- stant in most patients, but there was progression in 15 patients (4 with bicuspid aortic valves and 11 with tricuspid aortic valves). This includes the 4 patients who underwent subsequent reoperation for significant recurrent aortic insufficiency.

Survival at 1,3 , and 5 years was $100 \%$ in group A and $93 \%, 87 \%$, and $82 \%$ in group $\mathrm{B}$, respectively $(P=.004)$. There was no episode of thromboembolism or endocarditis. Reoperation for recurrent aortic insufficiency was necessary in 1 patient in group A (7 months) and 3 patients in group B (2, 4, and 68 months postoperatively, respectively). The cause of recurrent regurgitation in the patient from group A was dilatation at the aortoventricular level. The aortoventricular dimensions remained constant in all other patients. In group B the causes for reoperation were progressive aortoventricular dilatation $(\mathrm{n}=1)$, failure of paracommissural cusp plication $(\mathrm{n}=1)$, and secondary cusp retraction $(\mathrm{n}=1)$. The reoperations consisted of aortic valve replacement $(n=3)$ within the graft; in one patient the root was re-replaced with a stentless xenograft.

Mean systolic gradients at discharge were comparable (group A, $4.7 \pm 2.4 \mathrm{~mm} \mathrm{Hg}$; group B, $4.1 \pm 2.3 \mathrm{~mm} \mathrm{Hg}$ ). These gradients remained constant during the follow-up period and at 1 year (group A, $4.8 \pm 2.1 \mathrm{~mm} \mathrm{Hg}$; group B, $4.0 \pm 2 \mathrm{~mm} \mathrm{Hg}$ ), and they were $4.5 \pm 2.3 \mathrm{~mm} \mathrm{Hg}$ in group A and $3.9 \pm 2.2 \mathrm{~mm} \mathrm{Hg}$ in group B at 5 years.

Actuarial freedom from aortic regurgitation of grade II or higher at 1, 3, and 5 years was $98 \%, 96 \%$, and $96 \%$, respectively, in group A. In group B this was $93 \%, 89 \%$, and $83 \%(P=.07$; Figure 1$)$. Freedom from reoperation at 1,3 , and 5 years was $98 \%$ in group A and in group B $(P=$ .73; Figure 2). 


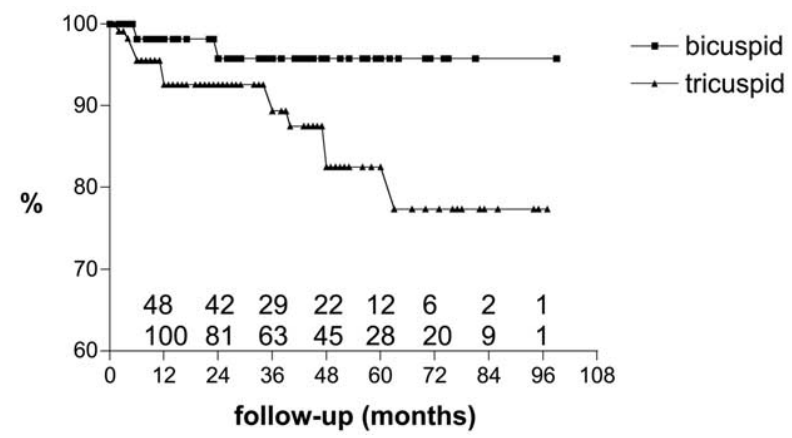

Figure 1. Actuarial freedom from aortic regurgitation of II or greater after root remodeling in the presence of bicuspid or tricuspid aortic valve anatomy.

\section{Discussion}

The current analysis deals with essentially 2 questions: whether bicuspid valves should be repaired and how to deal with the coexistence of aortic dilatation. A bicuspid aortic valve occurs in $1 \%$ to $2 \%$ of the general population. ${ }^{21}$ It is currently seen in more than $35 \%$ of patients referred for aortic valve surgery, ${ }^{22}$ indicating the high potential of this valve anatomy to exhibit dysfunction. It has been hypothesized that all bicuspid aortic valves will ultimately experience morphologic stenosis as a response to abnormally high mechanical cusp stress. ${ }^{23}$ Other observations indicate that bicuspid valves might take different natural courses depending on morphologic differences. ${ }^{21,24}$ Calcification and stenosis seem to affect bicuspid aortic valves that have no redundant cusp tissue and affect individuals at an average age of 61 years. $^{21}$ Bicuspid aortic valves with redundant cusp tissue frequently have prolapse of the larger (congenitally fused) cusp, resulting in regurgitation. ${ }^{24}$ Calcification is rare, and the patients become symptomatic between the second and sixth decade of life, with a mean age of 31 years. $^{21,24}$ Bicuspid aortic valves can function without major hemodynamic abnormality up to the seventh decade of life. $^{25}$

The majority of patients with a regurgitant bicuspid aortic valve are young, with a high postoperative life expectancy. In these individuals the implantation of mechanical valves is associated with low linearized rates of valve thrombosis, thromboembolism, and hemorrhage. ${ }^{8}$ The high life expectancy will nevertheless lead to a considerable cumulative risk of valve-related morbidity. Biologic valve substitutes continue to be associated with an increased tendency toward degeneration. ${ }^{26}$ The pulmonary autograft appears to be an attractive alternative. However, there have been concerns over inherent structural wall abnormalities in the pulmonary trunk, potentially leading to dilatation and an increased failure rate of the autograft. ${ }^{7}$

Repair appears to be a potentially attractive alternative to valve replacement, at least if durability exceeds that of

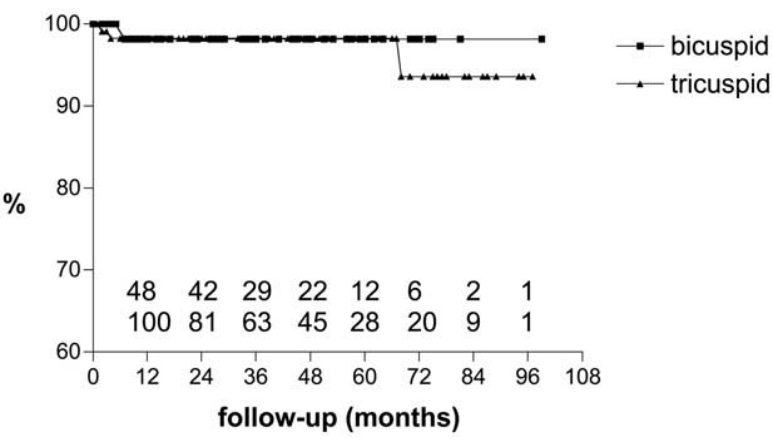

Figure 2. Actuarial freedom from reoperation for aortic valve dysfunction after root remodeling in the presence of bicuspid or tricuspid aortic valve anatomy.

biovalves. In analogy to the experience with mitral repair, it is hoped or expected that repair can minimize the incidence of valve-related morbidity, including the risk of endocarditis. Results of repair in regurgitant tricuspid aortic valves have not been uniformly good, most likely because of the high level of surgical judgment necessary for reconstruction of a valve with 3 coaptation lines. ${ }^{10}$ Reconstruction of bicuspid aortic valves was propagated by Cosgrove and coworkers $^{9}$ using a systematic repair approach. The early results were excellent, and there was minimal valve-related morbidity. Reconstructed bicuspid valves have shown a hemodynamic performance at rest and under exercise superior to that of prosthetic valves. ${ }^{27}$

In some series, however, valve stability was suboptimal, with a considerable rate of early and midterm failures. ${ }^{11}$ Also, in a later report of the initial group, ${ }^{12} 5$-year freedom from reoperation was only $87 \%$. The development of stenosis and recurrent regurgitation were observed as the 2 main reasons for reoperation. ${ }^{12}$ We have not yet seen increasing systolic gradients or the development of relevant stenosis, indicating a minimal chance of this valve becoming stenotic over an 8-year interval. Further observation will be necessary to obtain 10- and 15-year data.

Although triangular resection of cusp tissue appeared to be a risk factor for repair failure in one analysis, ${ }^{12}$ progressive aortic root enlargement was looked for and identified as a risk factor for recurrent regurgitation in at least one series. ${ }^{11}$ Although an association between presence of a bicuspid aortic valve and dilatation of the ascending aorta has been recognized, ${ }^{13,14}$ its exact cause is still unclear. It has been assumed that regurgitation or abnormal systolic flow through a stenotic bicuspid valve leads to aortic dilatation as a physiologic phenomenon. ${ }^{28}$ Previous studies, however, have demonstrated histopathologic changes in the ascending aortas of patients with bicuspid valves similar to those of cystic medial necrosis. ${ }^{15}$ Dilatation also occurs in patients with bicuspid aortic valves in the absence of stenosis or incompetence. ${ }^{13}$ In addition, aortic dilatation has 
been shown to progress after valve replacement (ie, elimination of the source of abnormal systolic and diastolic flow). ${ }^{29}$ The prognostic implications of aortic dilatation in the presence of a bicuspid aortic valve are increasingly recognized. ${ }^{16}$

If root enlargement is already present at the time of surgical intervention, the dilated parts of the aorta have to be replaced apart from the need of valve repair. Valve-preserving operations not only eliminate dilatation and improve coaptation of the aortic cusps but might also stabilize the valve repair, similar to the use of a ring in mitral reconstruction. The current results support this concept because midterm stability was better than that of isolated bicuspid valve repair. ${ }^{12}$ The combined approach did not lead to prolonged ischemic times if performed for bicuspid valve anatomy, and morbidity and mortality were low. Further comparison with patients undergoing remodeling in cases of tricuspid anatomy is limited by the fact that these 2 cohorts were not comparable regarding age and comorbidity. The tricuspid group was primarily used for comparison of valve function with an established concept. Repair stability was better in patients with bicuspid versus tricuspid anatomy, which is similar to other observations. ${ }^{10}$ Although the number of patients beyond the fifth postoperative year is still limited, 5- and 7-year freedom from reoperation was identical to that published for the pulmonary autograft. ${ }^{30}$

It is at this time unclear which patients should be treated with valve-preserving root replacement. Aortic dilatation is not invariably found in the presence of bicuspid valve anatomy. Normal or near-normal aortic dimensions have been found in more than $40 \%$ of patients. ${ }^{13,14}$ There are some patients who exhibit only a mild form of root dilatation, and the most extensive enlargement of aortic diameter is above the commissures. Some patients could also be treated with separate valve operations and supracommissural aortic replacement. We have arbitrarily chosen a diameter of the sinotubular junction of 33 to $35 \mathrm{~mm}$ (taking patient size into consideration) as the cutoff point for root replacement. This might seem aggressive, but it did not result in increased early morbidity and mortality compared with the results of valve repair in other series. ${ }^{9-12}$ Further experience and careful analysis will be necessary to better determine the size criteria for optimal choice of procedure. In older patients separate valve and aortic replacement or composite replacement with a bioprosthesis might be an alternative.

The type of valve-preserving procedure might be controversial. Remodeling of the aortic root ${ }^{1}$ and reimplantation of the aortic valve $e^{3}$ have been used to improve the geometry of the tricuspid aortic valve (ie, the symmetric root). Functionally, both approaches lead to similar results in tricuspid valve anatomy, with a freedom from reoperation in remodeling of $89 \%$ after 5 and 10 years and $85 \%$ after 15 years and a $99 \%$ freedom from reoperation at 5 and 8 years for reimplantation. ${ }^{2,4}$

In the presence of a bicuspid aortic valve, the anatomic characteristics of the valve and root have to be taken into consideration. The congenitally fused cusp is smaller than the sum of 2 cusps in a tricuspid valve and takes up approximately $55 \%$ of the root circumference compared with $67 \%$ in normal anatomy. Its insertion is higher than the insertion of the larger noncoronary cusp. The rudimentary commissure between the 2 parts of the fused cusp is lower than the 2 commissures of the noncoronary cusp. The depth of the sinuses differs accordingly, with the noncoronary sinus being unusually deep. It has been our belief that this asymmetry of the root can be better preserved with root remodeling than with valve reimplantation. We have attempted to normalize root dimensions without altering the asymmetry associated with bicuspid anatomy by nature rather than normalizing root geometry. For these reasons, we have thus far applied the reimplantation procedure only in one patient with a bicuspid valve, in whom fusion between 2 cusps extended for less than a centimeter. Secondary dilatation at the aortoventricular level as a potential disadvantage of the remodeling procedure has occurred only once in our experience.

Our results indicate that valve-sparing aortic replacement is feasible in patients with aortic dilatation and regurgitation caused by a bicuspid valve. Postoperative systolic function of bicuspid valves is similar to that of normal tricuspid anatomy. Midterm follow-up shows no significant difference in valve stability between these 2 anatomic variants; if anything, repair is more durable in bicuspid aortic valves. The risk of reoperation is low. If necessary, a stented valve can easily be placed within the root graft. A more liberal use of valve preservation in patients with bicuspid aortic valves appears justified.

\section{References}

1. Sarsam M, Yacoub M. Remodeling of the aortic valve annulus. J Thorac Cardiovasc Surg. 1993;105:435-8.

2. Yacoub M, Gehle P, Chandrasekaran V, Birks E, Child A, RadleySmith R. Late results of a valve-preserving operation in patients with aneurysms of the ascending aorta and root. J Thorac Cardiovasc Surg. 1998;115:1080-90.

3. David TE, Feindel CM, Bos J. Repair of the aortic valve in patients with aortic insufficiency and aortic root aneurysm. J Thorac Cardiovasc Surg. 1995;109:345-51.

4. David TE, Ivanov J, Armstrong S, Feindel C, Webb GD. Aortic valve-sparing operations in patients with aneurysms of the aortic root or ascending aorta. Ann Thorac Surg. 2002;74:1758-61.

5. Schäfers H-J, Fries R, Langer F, Nikoloudakis N, Graeter T, Grundmann U. Valve-preserving replacement of the ascending aorta: remodeling versus reimplantation. J Thorac Cardiovasc Surg. 1998;116: 990-6.

6. Langer F, Graeter T, Nikoloudakis N, Aicher D, Wendler O, Schäfers HJ. Valve-preserving aortic replacement: does the additional repair of leaflet prolapse adversely affect the results? J Thorac Cardiovasc Surg. 2001;122:270-7. 
7. De Sa M, Moshkovitz Y, Butany J, David TE. Histologic abnormalities of the ascending aorta and pulmonary trunk in patients with bicuspid aortic valve disease: clinical relevance to the Ross procedure. J Thorac Cardiovasc Surg. 1999;118:588-96.

8. Hagl C, Strauch JT, Spielvogel D, Galla JD, Lansman SL, Squitieri R, et al. Is the Bentall procedure for ascending aorta or aortic valve replacement the best approach for long-term event-free survival? Ann Thorac Surg. 2003;76:698-703.

9. Cosgrove DM, Rosenkranz ER, Hendren WG, Bartlett JC, Stewart WJ. Valvuloplasty for aortic insufficiency. J Thorac Cardiovasc Surg. 1991;102:571-7.

10. Haydar HS, He G-W, Hovaguimian H, McIrvin DM, King, DH, Starr A. Valve repair for aortic insufficiency: surgical classification and techniques. Eur J Cardiothorac Surg. 1997;11:258-65.

11. Moidl R, Moritz A, Simon P, Kupilik N, Wolner E, Mohl W. Echocardiographic results after repair of incompetent bicuspid aortic valves. Ann Thorac Surg. 1995;60:669-72.

12. Casselman FP, Gilinov A, Akhrass R, Kasirajan V, Blackstone E, Cosgrove D. Intermediate-term durability of bicuspid aortic valve repair for prolapsing leaflet. Eur J Cardiothorac Surg. 1999;15:302-8.

13. Nistri S, Sorbo MD, Marin M, Palisi M, Scognamiglio R, Thiene G. Aortic root dilatation in young men with normally functioning bicuspid aortic valves. Heart. 1999;82:19-22.

14. Nkomo VT, Enriquez-Sarano M, Ammash NM, Melton LJ 3rd, Bailey KR, Desjardins V, et al. Bicuspid aortic valve associated with aortic dilatation: a community-based study. Arterioscler Thromb Vasc Biol. 2003;23:351-6.

15. Bonderman D, Gharehbaghi-Schnell E, Wollenek G, Maurer G, Baumgartner H, Lang IM. Mechanisms underlying aortic dilatation in congenital aortic valve malformation. Circulation. 1999;99:2138-43.

16. Lung B, Gohlke-Bärwolf $\mathrm{C}$, Tornos $\mathrm{P}$. Recommendations on the management of the asymptomatic patient with valvular heart disease. Eur Heart J. 2002;23:1253-66.

17. Schäfers H-J, Langer F, Aicher D, Graeter T, Wendler O. Remodeling of the aortic root and reconstruction of the bicuspid aortic valve. Ann Thorac Surg. 2000;70:542-6.

18. Masuyama TK, Kodama AK, Kitabatake A. Non-invasive evaluation of aortic regurgitation by continuous-wave Doppler echocardiography. Circulation. 1987;73:460-6.

19. Galassi AR, Nihoyannopoulos P, Pupita G, Odwadara H, Crea F, McKenna WJ. Assessment of color flow imaging in the grading of valvular regurgitation. Eur Heart J. 1990;11:1101-8.

20. Skjerpe T, Hegrenaes L, Hatle L. Noninvasive estimation of valve area in patients with aortic stenosis by Doppler ultrasound and two-dimensional echocardiography. Circulation. 1985;72:810-8.

21. Roberts WC. The congenitally bicuspid aortic valve: a study of 85 autopsy cases. Am J Cardiol. 1970;26:72-83.

22. Thubrikar M. The aortic valve. Boca Raton (FL): CRC Press; 1990. p. 158-9.

23. Robicsek F, Thubrikar MJ, Cook JW, Fowler B. The congenitally bicuspid aortic valve: how does it function? Why does it fail? Ann Thorac Surg. 2004;77:177-85.

24. Olson LJ, Subramanian R, Edwards WD. Surgical pathology of pure aortic insufficiency: a study of 225 cases. Mayo Clin Proc. 1984;59: 835-41.

25. Fenoglio J, McAllister H, DeCastro CD, Davia J, Cheitlin M. Congenital bicuspid aortic valve after the age 20. Am J Cardiol. 1977;39: 164-9.

26. Jamieson WRE, Rosado LJ, Munro AI. Carpentier-Edwards standard porcine bioprothesis: primary tissue failure (structural valve deterioration) by age groups. Ann Thorac Surg. 1988;46:155-62.

27. Graeter TP, Kindermann M, Fries R, Langer F, Schäfers HJ. Comparison of aortic valve gradient during exercise after aortic valve reconstruction. Chest. 2000;118:1271-7.

28. Holman E. The obscure physiology of post-stenotic dilatation: its relation to the development of aneurysms. J Thorac Surg. 1954;28: 109-33.

29. Yasuda H, Nakatani S, Stugaard M, Tsujita-Kuroda Y, Bando K, Kobayashi J, et al. Failure to prevent progressive dilation of ascending aorta by aortic valve replacement in patients with bicuspid aortic valve: comparison with tricuspid aortic valve. Circulation. 2003;108(suppl II):II291-4.

30. Paparella D, David TE, Armstrong S, Ivanov J. Mid-term results of the Ross procedure. J Card Surg. 2001;16:338-43.

\section{Discussion}

Dr Christopher Feindel (Toronto, Ontario, Canada). Dr Aicher, thank you very much for a very clear presentation. You obviously have some excellent results from your group.

I think many of us are still concerned about sparing a bicuspid aortic valve, but I think your results thus far strongly suggest that we should seriously consider doing that. However, at the same time, I think it is extremely important that you, as you have been, continue to follow these patients and give us a report down the road to see how they are doing.

I have a couple of questions, and the first question relates to the remodeling operation that was described, as you know, by Yacoub. You are using that operation rather than the reimplantation operation, as described by Dr David and myself, and I swear Dr David did not prompt me to ask this question. As you know, the remodeling operation, at least in our experience, tended not to prevent the gradual and increasing aortic root or aortic annular dilatation, and we found that those patients ended up with more aortic insufficiency than with the reimplantation operation.

In your series you had only one case in which the regurgitation developed in the valve, and I am wondering whether there was any reason why the failure was so low. Were there other things that you were using to protect the annulus from dilating?

Dr Aicher. We have treated true annular ectasia with the reimplantation technique for 8 years, but since that time, we have had only one patient with a bicuspid aortic valve, and this patient had only a limited fusion of the cusps and almost normal geometry. Therefore in the series I presented here, we had a few patients who had aortoventricular diameters of close to $30 \mathrm{~mm}$, and in those patients we added subcommissural plicating sutures, such as the Cabrol type, to the repair for additional stabilization. Overall, we have only seen limited secondary dilatations, and until now, we do not know whether dilatation of the aortoventricular junction is a real problem in patients with bicuspid aortic valve disease.

Dr Feindel. Thank you. The second question really relates to whether you learned anything by looking at these valves as far as deciding which valve to spare. Surgeons that start to embark on these operations, I think, will find that the most difficult question is to try and decide which valve should be spared and which should not be spared. When you looked at these bicuspid valves, were there any criteria that you used to make that decision, and if so, would you be prepared to share those with us?

Dr Aicher. I agree with all your considerations that preservation of the valve is a decision of judgment and experience that can only be made by an experienced surgeon. Therefore we do correct prolapses present in 1 or 2 cusps, but prolapse in 3 cusps is very difficult to judge because you have no normal reference point. Therefore we would go for replacement in a valve of 3-leaflet prolapse. Another exclusion criterion for us is severe calcification of the cusp. Overall, we believe that it is easier to preserve a bicuspid aortic valve with an easy geometry and only one coaptation line than a tricuspid aortic valve with 3 coaptation lines. 
Dr Feindel. Thank you very much. We look forward to hearing future follow-up of these patients.

Dr Meong G. Song (Seoul, Korea). Congratulations on a nice presentation, but I have a couple of questions. Basically, a bicuspid aortic valve is an obstructive condition of the aortic valve instead of being a disease of regurgitation. Therefore we should be more concerned about aortic stenosis. Your technique of plication or triangular resection might actually aggravate aortic stenosis. Bearing this in mind, I have a couple of questions.

Did you check or measure the peak gradient across the aortic valve preoperatively and postoperatively? And did you measure the effective orifice area?

Also, the sinotubular junction area changes a huge amount throughout the cardiac cycle, as much as $63 \%$ or more. How then did you decide on the diameter of the graft, because if you choose one with a smaller diameter, it might cause some restriction of the sinotubular junction, aggravating aortic stenosis? Of course, patients will survive, but some degree of aortic stenosis will remain.

Dr Aicher. here is one hypothesis that says that all bicuspid aortic valves ultimately have aortic valve stenosis, but there are other considerations that show that bicuspid aortic valves can take different natural courses depending on morphologic differences. Therefore we know that calcification and stenosis occurs in patients who have no redundant cusp tissue, and this affects patients at an older age of about 60 years, but patients with bicuspid aortic valves who have redundant cusp tissue normally experience aortic regurgitation, and this affects the patient at a much younger age, at about an average age of 30 years.

Therefore in our series, we did not see any relevant postoperative systolic gradient or stenosis. I think the risk is low that patients will have stenosis after that operation.

Dr Song. Did you measure the effective orifice area?

Dr Aicher. No, we did not measure the orifice area. We only measured the systolic gradient.

Dr Song. If you measured only the mean gradient, it does not tell you anything. The peak gradient is more important. Also, you should measure the effective orifice area. It is more important.

Dr Aicher. We did not only measure the mean but also the peak gradients, and they were not increased after the operation.

The Journal of Thoracic and Cardiovascular Surgery Conflict of Interest Policy

To assure fairness to authors submitting work for consideration in The Journal of Thoracic and Cardiovascular Surgery, a mechanism exists for managing conflicts of interest. The editor and each of the section editors complete a "Conflict of Interest" form that identifies any and all relationships with commercial and other academic entities. When the editor has a potential conflict because of a relationship with another entity or author, the editor appoints an alternate editor from among the section editors or editorial board members who assumes the entire responsibility for final decisions on the manuscript in question. The editor does not read the reviews that are submitted nor engage in discussing the manuscript prior to the final decision. When the conflict of interest involves a section editor, a "guest section editor" is appointed who fills the role normally played by the conflicted section editor. All members of the editorial board and reviewers are asked to indicate any conflict of interest when they agree to review a manuscript. 